\title{
Effect of Proton Conduction on the Charge Storage Mechanism of a MOF as Supercapacitor Electrode
}

\author{
Ling-Di Ye, ${ }^{\dagger a}$ Farzaneh Rouhani, ${ }^{\text {tb }}$ Hamed Kaviani, ${ }^{\text {tb }}$ Qian Miao, ${ }^{\dagger a}$ Xiao-Qing Cai, ${ }^{* a}$ Ali Morsali, ${ }^{* b}$ \\ Mao-Lin $\mathrm{Hu} *$ a \\ ${ }^{a}$ College of Chemistry and Materials Engineering, Wenzhou University, Wenzhou 325035, P. R. \\ China. \\ b. Department of Chemistry, Faculty of Sciences, Tarbiat Modares University, Tehran 14115- \\ 175, Iran. \\ $\dagger$ Authors contributed equally
}

\section{Materials and methods}

All raw materials were purchased from Merck and Aldrich and were used without further purification. The IR spectra (in $\mathrm{KBr}$ ) have been recorded by Nicolet Fourier Transform IR, Nicolet 100 spectrometer in the range of $4000-400 \mathrm{~cm}^{-1}$. Thermogravimetric analysis (TGA) of the TMU-60 was recorded by computer-controlled PL-STA 1500 apparatus from 25 to 600 ${ }^{\circ} \mathrm{C}$ into alumina pans with the gradient of $10{ }^{\circ} \mathrm{C} /$ min under neutral $\mathrm{N}_{2}$ atmosphere. The crystallographic measurements of TMU-60 were performed by the Bruker APEX area-detector diffractometer. The intensity data were collected using graphite monochromated Mo-K $\alpha$ radiation $(\lambda=0.71073 \AA)$ at $298 \mathrm{~K}$. The X-ray powder diffraction (XRD) measurements were done by using a Philips X'pert diffractometer with monochromated $\mathrm{Cu}-\mathrm{k} \alpha$ radiation $(\lambda=1.54056 \mathrm{~A})$. The adsorption/desorption isotherm of $\mathrm{N}_{2}$ was measured at liquid nitrogen temperature (77 K) using a Micromeritics ASAP 2020 analyzer. The specific surface area was calculated by the Brunauer-Emmett-Teller (BET) method. All electrochemical tests were performed on a EG\&G potentiostat/galvanostat electrochemical workstation. 
Table S.1. Crystal and structure refinement data of TMU-60.

\begin{tabular}{|c|c|c|c|}
\hline Identification code & TMU-60 & $\alpha(\mathrm{deg})$ & 90 \\
\hline Chemical formula & ${\mathrm{Zn} \mathrm{C}_{21} \mathrm{H}_{15} \mathrm{~N}_{2} \mathrm{O}_{5}}$ & $\beta(\mathrm{deg})$ & 90 \\
\hline $\begin{array}{c}\text { computing_structure_re } \\
\text { finement }\end{array}$ & SHELXL-2016/6 & $\gamma(\mathrm{deg})$ & 90 \\
\hline $\begin{array}{c}\text { Chemical formula } \\
\text { weight }\end{array}$ & 420.38 & $\mathrm{~V}\left(\dot{\mathrm{A}}^{3}\right)$ & 16 \\
\hline $\mathrm{T}(\mathrm{K})$ & 293.2 & $\mathrm{Z}$ & 3600 \\
\hline $\begin{array}{c}\text { Crystal syst } \\
\text { Space group }\end{array}$ & Orthorhombic & $\mathrm{F}(000)$ & 0.0448 \\
\hline $\mathrm{a}\left({ }^{\circ} \mathrm{A}\right)$ & $17.6350(15)$ & Goodness-of-fit on $F^{2}$ & 1.103 \\
\hline $\mathrm{b}\left({ }^{\circ} \mathrm{A}\right)$ & $22.345(2)$ & wR factor & 0.1080 \\
\hline $\mathrm{c}\left({ }^{\circ} \mathrm{A}\right)$ & $22.944(2)$ & CCDC number & 1862166 \\
\hline
\end{tabular}

Table S.2. Selected bond lengths $(\dot{\mathrm{A}})$ and angles $\left({ }^{\circ}\right)$ for TMU-60.

\begin{tabular}{|c|c|c|c|}
\hline \multicolumn{3}{|c|}{ Bond Angle } \\
\hline $\mathrm{Zn}_{1}-\mathrm{O}_{3}$ & $2.046(2)$ & $\mathrm{C}_{17}-\mathrm{C}_{19}-\mathrm{C}_{20}$ & 122.34 \\
\hline $\mathrm{Zn}_{1}-\mathrm{N}_{1}$ & $2.026(2)$ & $\mathrm{C}_{19}-\mathrm{C}_{20}-\mathrm{C}_{20}$ & 124.80 \\
\hline $\mathrm{C}_{20}-\mathrm{C}_{20}$ & $1.371(3)$ & $\mathrm{C}_{20}-\mathrm{C}_{20}-\mathrm{N}_{2}$ & 120.65 \\
\hline $\mathrm{C}_{20}-\mathrm{N}_{2}$ & $1.391(3)$ & $\mathrm{C}_{20}-\mathrm{N}_{2}-\mathrm{C}_{21}$ & 116.34 \\
\hline $\mathrm{C}_{21}-\mathrm{N}_{2}$ & $1.438(4)$ & $\mathrm{N}_{2}-\mathrm{C}_{21}-\mathrm{C}_{21}$ & 110.70 \\
\hline $\mathrm{C}_{21}-\mathrm{C}_{21}$ & $1.496(8)$ & $\mathrm{C}_{7}-\mathrm{O}_{5}-\mathrm{C}_{8}$ & 118.50 \\
\hline $\mathrm{N}_{2}-\mathrm{H}_{2}$ & $0.820(3)$ & $\mathrm{C}_{6}-\mathrm{C}_{7}-0 \mathrm{O}_{5}$ & 122.95 \\
\hline
\end{tabular}




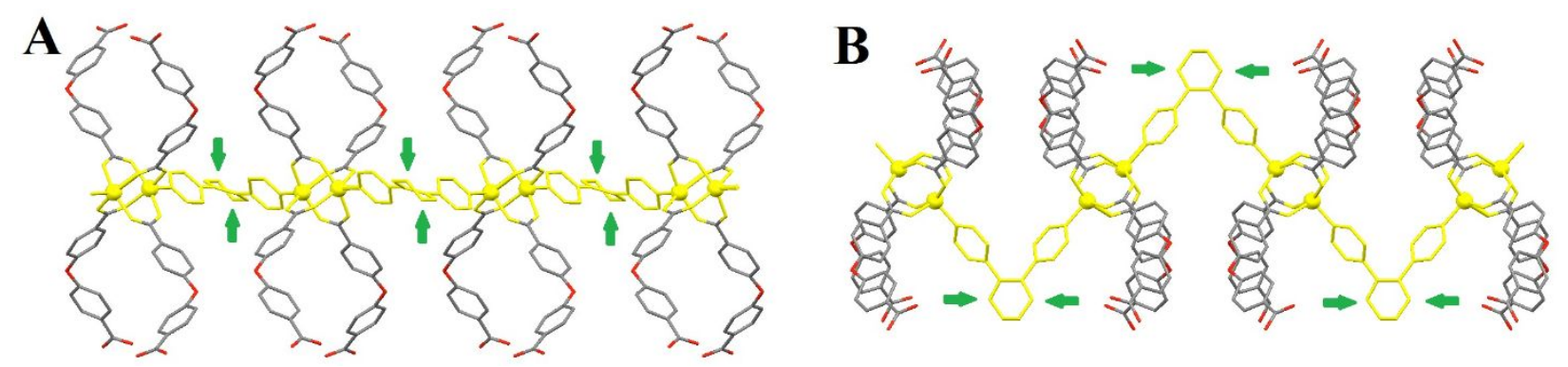

Figure S1. Schematic figure of amine orientation of TMU-60, along the axis a (A) and axis b (B).

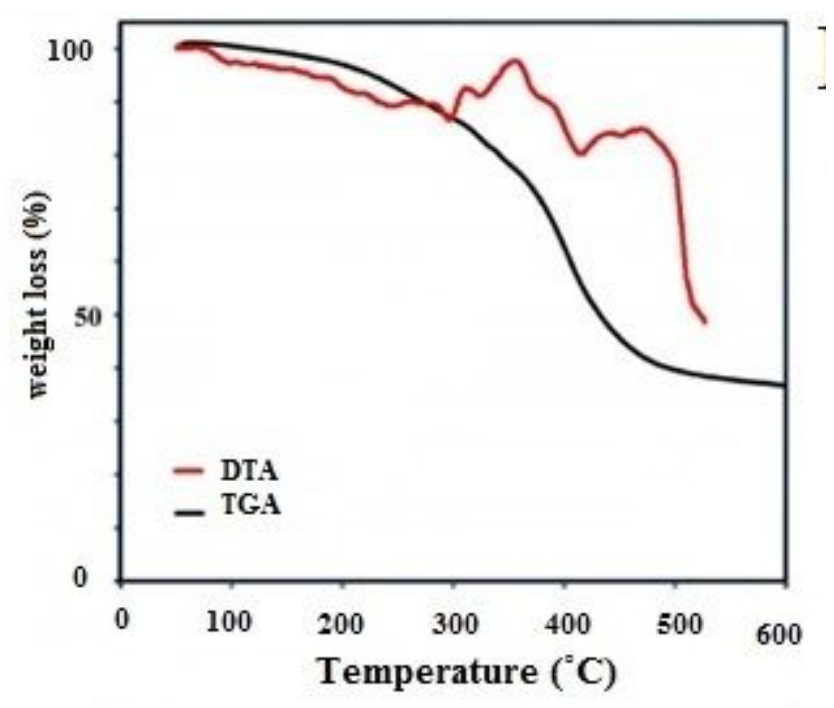

Figure S2. TGA analysis of non-activated TMU-60 at $5{ }^{\circ} \mathrm{C} / \mathrm{min}$.

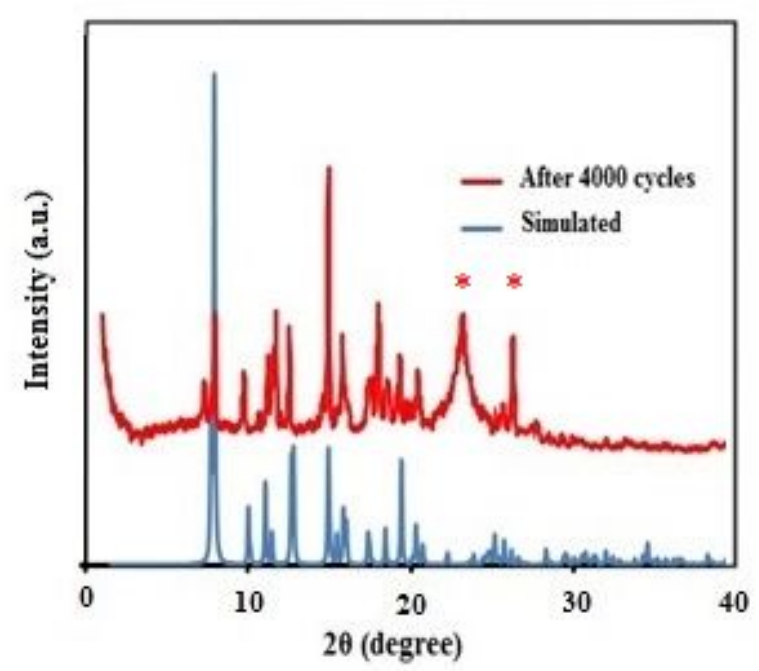

Figure S3. XRD pattern of TMU-60 after 4000 cycle of charge/discharge. 